\title{
Copenhagen Index versus ROMA in the preoperative ovarian malignancy risk stratification: result from the first Vietnamese prospective cohort study
}

\section{Doan Tu Tran}

Hue University of Medicine and Pharmacy

\section{Van Khoa Vo}

Hue University of Medicine and Pharmacy

Minh Tam Le

Hue University of Medicine and Pharmacy https://orcid.org/0000-0001-6225-3108

\section{Linus Chuang}

Nuvance Health, University of Vermont Larner College of Medicine

Vu Quoc Huy Nguyen ( $\nabla$ nvqhuy@huemed-univ.edu.vn )

Hue University of Medicine and Pharmacy https://orcid.org/0000-0002-4744-7059

\section{Research Article}

Keywords: Ovarian tumor, Copenhagen Index (CPH-I), Risk of Ovarian Malignancy Algorithm (ROMA), CA125, HE4

Posted Date: March 30th, 2021

DOl: https://doi.org/10.21203/rs.3.rs-366745/v2

License: (1) This work is licensed under a Creative Commons Attribution 4.0 International License. Read Full License 


\section{Abstract}

Objectives: This study aimed to evaluate the diagnostic performances of the Copenhagen Index (CPH-I) and Risk of Ovarian Malignancy Algorithm (ROMA) in the preoperative prediction of ovarian cancer.

Methods: In a prospective cohort study, data were collected from 475 patients with ovarian masses who were hospitalized at the Departments of Obstetrics and Gynecology, Hue Central Hospital and Hue University of Medicine and Pharmacy Hospital, Vietnam, between January 2018 and June 2020. ROMA and $\mathrm{CPH}-\mathrm{I}$ were included for the women who had measurements of serum carbohydrate antigen (CA-125) and human epididymis protein (HE4). Matching these values to postoperative histopathology resulted in the preoperative prediction values. The final diagnosis was based on clinical features, histologic and radiologic findings, and the International Federation of Gynecology and Obstetrics (FIGO) 2014 stages of ovarian cancer were recorded.

Results: Among the 475 women, 408 had benign tumors, 5 had borderline tumors and 62 had malignant tumors. The two indices showed similar discriminatory performances with no significant differences $(p>$ 0.05). At an optimal cut-off, the sensitivities/specificities of ROMA and CPH-I for ovarian cancer diagnosis were $76.1 \%$ and $87.0 \%, 83.6 \%$ and $78.7 \%$, respectively. The optimal cut-off for $\mathrm{CPH}-\mathrm{I}$ was $1.89 \%$. The areas under the ROC curves (AUCs) of ROMA and CPH-I were 0.860 (95\% Cl: $0.825-0.890)$ and $0.868(95 \% \mathrm{Cl}: 0.833-0.896)$, respectively.

Conclusions: The introduction of the Copenhagen Index to help stratify the malignancy risk of ovarian tumors, irrespective of menopausal status, might be applied as a simple alternative with a similar efficacy to ROMA in clinical practice.

\section{Introduction}

Ovarian cancer $(\mathrm{OC})$ is one of the ten most commonly diagnosed cancers in women and has the highest mortality rate and the worst prognosis of all gynecological cancers (1). In 2018, 295,414 cases of OC were detected in the world, and 184,799 died, with the highest incidence in developed countries (2). In 2019, approximately 22,530 cases of OC were diagnosed in the United States, ranking second in pelvic cancers after cancer of the uterus, of which there were 13,980 deaths (3). The mortality rate has not changed in the past 30 years, and it is predicted that by 2040 , this rate will be significantly increasing $(4,5)$. Since $70 \%$ of ovarian cancers are diagnosed in an advanced stage (stage III - IV), when the disease has spread to the pelvic and abdominal region, the 5 -year survival rate is $20-25 \%$, but if detected in the early stage, this rate is increased to $90 \%(4,6)$. Therefore, early detection has important implications for the treatment, quality of life and prognosis of patients $(7,8)$.

In the United Kingdom Collaborative Trial of Ovarian Cancer Screening (UKCTOCS), there were 78 ovarian cancer cases in 1,590 adnexal tumors, which were detected after screening 48,230 women by transvaginal ultrasound and 50,078 women using biochemical markers (CA-125, HE4) (9). In 2009, Moore et al. developed the Risk of Ovarian Malignancy Algorithm (ROMA) by integrating serum CA-125 and HE4 
values and menopausal status to differentiate between low- and high-risk patients with OC (10). In 2015, Karlsen et al. developed the Copenhagen Index (CPH-I) based on these two biomarkers and patient age. The areas under the ROC curve (AUCs) that predicted OC by CPH-I and ROMA were 0.960 and 0.954 , respectively, thereby showing that the values of these two indicators were equal. The Copenhagen Index has the advantage of not depending on ultrasound and menopausal status, and the age variable is easy to collect, simple, and objective (11). Therefore, the Copenhagen Index's advent promises to be a reliable, objective, and widely applied tool at the grassroots level.

The purpose of this study was to compare the Copenhagen Index and the ROMA in the preoperative prediction of ovarian cancer.

\section{Methods}

The study was conducted on women diagnosed with ovarian tumors who were hospitalized at the Departments of Obstetrics and Gynecology, Hue Central Hospital and Hue University of Medicine and Pharmacy Hospital, Vietnam, between January 2018 and June 2020.

The sample size was calculated according to the formula to estimate the specificity in two steps:

Step 1: Calculate FP + TN

$$
F P+T N=\frac{Z_{\frac{\alpha}{2}}^{2} x p_{s p} x\left(1-p_{s p}\right)}{w^{2}}
$$

Step 2: Calculate sample size

$$
N_{s p}=\frac{F P+T N}{1-p_{d i s}}
$$

Abbreviations: FP, False Positive; TN, True Negative, $z_{\alpha / 2}^{2}=1.96$ with $\alpha=0.05 ; w=0,04$. The $p_{s p}$ value from the study of Adriana Yoshida (2016) is 0.844 (12). According to GLOBOCAN (2018), the prevalence rate of $\mathrm{OC}$ in Vietnam ( $\mathrm{p}_{\text {dis }}$ ) is 7.67 cases $/ 100,000$ women $=0.000767$ (2). The minimum sample size $\left(N_{s p}\right)$ calculated by the formula was 317 patients. At the end of the study, 475 women who met the disease selection criteria were included.

In this prospective cohort study, the research steps included administrative interviews, medical history, physical examination and quantitative assays of serum CA-125 and HE4. At Hue University of Medicine and Pharmacy Hospital, serum CA-125 and HE4 tests were conducted using an electrochemiluminescence immunoassay on the COBAS 6000 system, Roche, Switzerland. Test results 
were controlled by the Internal Quality Control (IQC) system with RANDOX's standard control samples and programs. Calibration samples were performed daily on the system of testing machines before being tested. At Hue Central Hospital, tests for CA-125 and HE4 were conducted by chemiluminescent microparticle immunoassay on an Architect 11000 system (Abbott Diagnostics). The tests were quality checked daily (internal inspection) and were subjected to external inspection at the Ho Chi Minh City Standardization Center.

The Copenhagen Index predicts the risk of a preoperative ovarian tumor malignancy according to the algorithm below (11):

$\mathrm{CPH}-\mathrm{I}=-14,0647+1,0649 * \log _{2}(\mathrm{HE} 4)+0,6050 * \log _{2}(\mathrm{CA}-125)+0,2672 *$ Age $/ 10$

$\mathrm{PP}=\mathrm{e}^{(\mathrm{CPH}-\mathrm{I})} /\left(1+\mathrm{e}^{(\mathrm{CPH}-\mathrm{I})}\right)$

The ROMA index was calculated to predict the risk of ovarian tumor malignancy before surgery according to the following algorithm: ROMA $(\%)=\exp (\mathrm{PI}) /[1+\exp (\mathrm{PI})] * 100$

$\mathrm{PI}$ is the prediction index, determined as follows:

- Premenopausal women: $\mathrm{Pl}=-12,0+2,38 * \operatorname{Ln}[\mathrm{HE} 4]+0,0626 * \mathrm{Ln}[\mathrm{CA}-125]$

- Postmenopausal women: $\mathrm{Pl}=-8,09+1,04 * \operatorname{Ln}[\mathrm{HE} 4]+0,732 * \operatorname{Ln}[\mathrm{CA}-125]$

The ROMA cut-off point values were applied according to the technical Instructions of the Cobas 6000 system and the ARCHITECT system. Patients are supposed to have a high risk of ovarian cancer when:

\begin{tabular}{|lll|}
\hline Test system & Pre-menopausal group & Post-menopausal group \\
\hline Cobas 6000 (ROMA 1) & $\geq 11,4 \%$ & $\geq 29,9 \%$ \\
Architect i1000 (ROMA 2) & $\geq 7,4 \%$ & $\geq 25,3 \%$ \\
\hline
\end{tabular}

The patients were then surgically managed and diagnosed with histopathology according to the World Health Organization (WHO) 2014 (13). Finally, the parameters were compared with the histopathological results (including benign and malignant ovarian tumors) to calculate and compare with the diagnostic values of $\mathrm{CPH}-\mathrm{I}$ and ROMA. Based on these two indicators, the patients were classified into the benign tumor group or malignant tumor group (borderline and malignant tumors).

\section{Statistical analysis}

Data analyses were performed using the statistical software SPSS 20.0 (SPSS, Inc., Chicago, IL, USA), and receiver operative curve (ROC) analysis was performed with MedCalc. Categorical variables were reported as numbers (percentages), and continuous variables were reported as medians (SDs, standard deviations; ranges). The chi-square test (c2) was used to evaluate intergroup differences, and $p$ 
$<0.05$ was considered significant. The Kruskal-Wallis test was used to compare the differences between three groups that were not normally distributed.

\section{Ethical Approval}

Ethical approval for the study protocol was by the Ethics Committee for Biomedical Research at Hue University of Medicine and Pharmacy, Hue, Vietnam (decision number H2018/359, issued on June 22, 2018). Informed consent was obtained from the study subjects.

\section{Results}

Of the 475 patients, 408,5 and 62 were diagnosed with benign tumors, borderline tumors, and OC, respectively. The main characteristics of individual patient subgroups according to histopathologic diagnosis are shown in Table 1. The mean age of women in the $\mathrm{OC}$ group was higher than that of women in the benign tumor group. There were significant differences in age, menopausal status, and marital status between the two groups $(p<0.05)$. The incidence of OC in the postmenopausal group was $59.7 \%$.

Histological classification of participants was demonstrated as follows: among the 408 women diagnosed with benign tumors, 171 (41.9\%) had mature cystic teratoma, $165(40.4 \%)$ had serous cystadenoma, and 37 (9.1\%) had endometrioses of the ovary. In the borderline tumor group, 4 of 5 patients had serous borderline tumors. In patients with OC, serous adenocarcinoma was seen in 27 cases (43.5\%), followed by $12(19.7 \%)$ with mucinous adenocarcinoma, $6(9.7 \%)$ with poorly differentiated carcinoma, and $6(9.7 \%)$ with dysgerminoma. Clinical staging was performed according to the International Federation of Gynecology and Obstetrics (FIGO): 19 (30.6\%) cases were stage I, 8 $(12.9 \%)$ cases were stage II, $26(41.9 \%)$ cases were stage III, and $9(14.5 \%)$ cases were stage IV.

The median values of $\mathrm{CPH}-\mathrm{I}$ and ROMA of the OC group were statistically higher than those of the benign tumor group (Kruskal - Wallis test) (Table 3). In the study sample, the median value of $\mathrm{CPH}-\mathrm{I}$ in the $\mathrm{OC}$ group was $24.81 \%$ (3.49-81.21\%), which was statistically higher than the value from the benign tumor group at $0.82 \%(0.44-1.76 \%)(p<0.05)$. The median ROMA value in the benign tumor group was $5.03 \%$ $(3.46-8.71 \%)$ and that of the OC group was $49.93 \%(12.78-81.22 \%)$; the difference was statistically significant $(p<0.05)$. The median values of the $\mathrm{CPH}-\mathrm{I}$ and ROMA of the postmenopausal group were higher than those of the premenopausal group, in both the $\mathrm{OC}$ group and the benign tumor group. Specifically, in the premenopausal group, the median values of $\mathrm{CPH}-\mathrm{I}$ for the $\mathrm{OC}$ group, benign tumor, and borderline tumor group were $4.87 \%(1.49-45.72 \%), 0.72 \%(0.41-1.43 \%)$ and $0.42 \%(0.29-$ $24.39 \%)$, respectively; the median values of ROMA for the OC group, benign tumor group, and borderline tumor group were $12.18 \%(6.11-62.06 \%), 4.58 \%$ (3.06 - 6.76\%) and 5.84\% (3.28-

$16.38 \%)$, respectively. For the postmenopausal subjects, the median values of $\mathrm{CPH}-\mathrm{I}$ for the OC group and benign tumor group were $45.49 \%(8.35-91.62 \%)$ and $1.49 \%(0.87-3.65 \%)$, respectively; the median values of ROMA for the OC group and benign tumor group were $72.37 \%(37.41-95.16 \%)$ and $10.59 \%$ (7.49-18.88\%), respectively. 
The values of the Copenhagen Index and the ROMA index in the prediction of OC risk before surgery are shown in Table 4 and Graph 1. In the study population, the AUCs of CPH-I and ROMA in the prediction of OC were equivalent, being $0.868(95 \% \mathrm{Cl}: 0.833-0.896)$ and $0.860(95 \% \mathrm{Cl}: 0.825-0.890)$, respectively. At the optimal cut-off point of $1.89 \%$, the Copenhagen Index had a sensitivity of $83.6 \%$ (95\% Cl: $72.5-$ $91.5 \%$ ) and specificity of $78.7 \%$ (95\% Cl: $74.4-82.6 \%$ ). With an optimal cut-off value of $12.1 \%$ for ROMA, the sensitivity and specificity were $76.1 \%(95 \% \mathrm{Cl}: 64.1-85.7 \%)$ and $87.0 \%(95 \% \mathrm{Cl}: 83.4-90.1 \%)$, respectively. The Copenhagen Index and the ROMA index are of equivalent value in the differential diagnosis of benign and malignant ovarian tumors; the difference was not statistically significant $(p>$ $0.05)$.

\section{Discussion}

The continuous variables ROMA and $\mathrm{CPH}-\mathrm{I}$ in this study have several nonstandard distributions. Therefore, the statistics of ROMA and CPH-I will be presented as median values (Q25\% - Q75\%).

The median values of $\mathrm{CPH}-\mathrm{I}$ and ROMA in the OC group, as shown in Table 3, were higher than those of the benign tumor group and borderline tumor group. Specifically, for the study population $(n=475)$, the median value of $\mathrm{CPH}-\mathrm{I}$ in the malignant group was $24.81 \%(3.49-81.21 \%)$, whereas in the benign tumor group, it was $0.42 \%(0.29-24.39 \%)$, and those in the borderline tumor group were $0.42 \%(0.29-$ $24.39 \%)$. The median values of ROMA in the cancer group, borderline group, and benign group were $49.93 \%$ (12.78 - 81.22\%), 5.48\% (3.28 - 16.38\%), and 5.03\% (3.46-8.71\%), respectively $(p<0.05)$. Compared to previous studies, the median values of $\mathrm{CPH}-\mathrm{I}$ and ROMA from our research are lower than those of some other studies in the world. According to Adriana Yoshida (2016), the median values of $\mathrm{CPH}-\mathrm{I}$ for benign tumors and ovarian carcinomas were $1.4 \%$ and $83.4 \%$, respectively (12). Meanwhile, in Lubos Minar's study, the median values of $\mathrm{CPH}-\mathrm{I}$ in the benign and malignant groups were $2.2 \%$ and $75.4 \%$, respectively (14) (Table 5). More detailed analysis in the premenopausal and postmenopausal groups, which examined the differences between $\mathrm{CPH}-\mathrm{I}$ and ROMA, showed that the median values of $\mathrm{CPH}-\mathrm{I}$ and ROMA were higher in the postmenopausal group than in the premenopausal group (Table 3). The median values of $\mathrm{CPH}-\mathrm{I}$ and ROMA in the postmenopausal group were higher than those in the premenopausal group. The sensitivity/specificity (Se/Sp) of $\mathrm{CPH}-\mathrm{I}$ in the absence of marginal ovarian tumors, nonepithelial OC, and OC metastasis was $89.7 \% / 85.3 \%$, but if the above objects were included, the corresponding Se/Sp became lower at $73.1 \% / 84.4 \%(12)$.

The Se/Sp of ROMA and CPH-I in the diagnosis of OC were $76.1 \% / 87.0 \%$ and $83.6 \% / 78.7 \%$, respectively. The optimal cut-off point of the $\mathrm{CPH}-\mathrm{I}$ was $1.89 \%$, and the AUCs of ROMA and $\mathrm{CPH}-\mathrm{I}$ were 0.860 (95\% Cl: $0.825-0.890)$ and 0.868 (95\% Cl: $0.833-0.896)$, respectively. Research by T. Nikola (2017) differential diagnosis between ovarian endometriosis and ovarian carcinoma showed that the accuracy of the Copenhagen Index was higher than that of ROMA, 93.75\% and $85.42 \%$, respectively (15).

Zhiheng Wang et al. (2019) argued that the HE4 level and ROMA and CPH-I values of epithelial ovarian cancer (EOC) stages I and II (I + II) were all higher than those of borderline ovarian tumor (BOT) stages I + 
II and benign groups in all premenopausal and postmenopausal groups $(p<0.01)$. When distinguishing BOT I+II from EOC I+II, the AUC-ROCs of CPH-I and HE4 were larger than that of CA-125 $(p<0.001)$. CPH-I is more valuable than CA-125 when distinguishing marginal ovarian tumors from stage I - II ovarian carcinoma, while HE4 may be better than CA-125 in the postmenopausal group; HE4 and CPH-I have been more advantageous than CA-125 when differentiating a borderline ovarian tumor from an early-stage ovarian carcinoma $(I+I)$ in the absence of histology or type of serum fluid. The AUCs of $\mathrm{CPH}-\mathrm{I}$ and ROMA in the premenopausal group were 0.779 and 0.760 , respectively, and those in the postmenopausal group were 0.802 and 0.774 , respectively. In the premenopausal group, the Se/Sp of ROMA and CPH-I were $78.69 \% / 64.75 \%$ and $70.49 \% / 78.69 \%$, respectively. In the postmenopausal group, the $\mathrm{Se} / \mathrm{Sp}$ of ROMA and $\mathrm{CPH}-\mathrm{I}$ were $82.98 \% / 68.18 \%$ and $85.11 \% / 68.18 \%$, respectively (16).

According to Estrid Høgdall, ROMA and $\mathrm{CPH}-\mathrm{I}$ can be used for the differential diagnosis between benign and malignant ovarian tumors [13]. Since family doctors might be unable to perform an ultrasound test, both ROMA and $\mathrm{CPH}-\mathrm{I}$ could provide the initial reliable information, which helps the patient obtain early diagnosis and proper treatment from specialized centers. In general, $\mathrm{CPH}-\mathrm{I}$ and ROMA have similar sensitivity and accuracy. $\mathrm{CPH}-\mathrm{I}$ is not identical to ROMA and RMI because it is not independent of ultrasound test and menopausal status. Menopausal status can be determined based on age, hormone concentration or amenorrhea per year, so the diagnosis of menopausal status has not been standardized. Therefore, CHP-I could be a simpler method to optimize management when assessing women with suspected OC, including age instead of menopausal status $(11,17)$.

The Copenhagen Index is a new indicator that has been introduced in several studies around the world. The ROMA algorithm is an index that the US Food and Drug Administration has introduced in clinical practice to distinguish benign and malignant ovarian tumors based on three variables: CA-125, serum HE4, and menopausal status (19). These two indexes have quite similar values since both are partially based on CA-125 and HE4. Since serum CA-125 and HE4 concentrations are affected by many factors, including age, smoking, uterine fibroids, pregnancy, endometriosis, pelvic inflammatory disease, and gallbladder stones, this will affect the values of the Copenhagen index and ROMA $(20,21)$. In the future, more research on these two indicators on different target groups will be conducted to clarify these differences, aiming to overcome the limitations of these indicators and improve clinical practice.

To the best of our knowledge, this is the first cohort study from Vietnam with a large number of ovarian tumor subjects included, examining the validity of $\mathrm{CPH}-\mathrm{I}$ and comparing it with those from ROMA in risk stratification for ovarian tumor malignancy. Although rigorously designed and implemented, the OC cases were still limited, and the laboratory equipment was different at the two facilities where the work was done, which could partially affect the homogeneity of the data analysis.

\section{Conclusions}

The introduction of the Copenhagen Index to help stratify the risk of ovarian tumor malignancy, irrespective of menopausal status, is similarly accurate to but simpler than ROMA and could therefore 
replace ROMA in clinical practice.

\section{Declarations}

\section{Author contributions}

TDT, LMT, LC, and NVQH conceived the study, coordinated its planning and implementation, and wrote the manuscript.

TDT, VVK, LMT coordinated data acquisition, participated in the data analysis and interpreted the results

LMT, LC and NVQH supervised the preparation and revision of the manuscript.

All authors have approved the submitted version of the manuscript.

\section{Acknowledgment}

None.

\section{Conflicts of interest}

The authors have no conflicts of interest.

\section{Funding}

Not applicable.

\section{Author information}

Tran Doan Tu: MD, MSc, Department of Obstetrics \& Gynecology, Hue University of Medicine and Pharmacy, Hue University, Hue 49120, Vietnam

Vo Van Khoa: MD, MSc, Department of Obstetrics \& Gynecology, Hue University of Medicine and Pharmacy, Hue University, Hue 49120, Vietnam

Le Minh Tam: M.D, PhD, Assoc. Professor, Department of Obstetrics and Gynecology, Hue University of Medicine and Pharmacy, Hue University, 06 Ngo Quyen Street, Hue 491200, Vietnam

Linus Chuang: MD, Professor, Obstetrics and Gynecology, Nuvance Health, University of Vermont Larner College of Medicine, United States

Nguyen Vu Quoc Huy: Professor, MD, PhD, Department of Obstetrics \& Gynecology, Hue University of Medicine and Pharmacy, Hue University, Hue 49120, Vietnam

\section{References}


1. Momenimovahed Z, Tiznobaik A, Taheri S, Salehiniya H. Ovarian cancer in the world: epidemiology and risk factors. Int J Women Health. 2019 Apr 30;11:287-99.

2. Bray F, Ferlay J, Soerjomataram I, Siegel RL, Torre LA, Jemal A. Global cancer statistics 2018 : GLOBOCAN estimates of incidence and mortality worldwide for 36 cancers in 185 countries. CA Cancer J Clin. 2018;68(6):394-424.

3. American Cancer Society. Cancer Facts \& Figures 2019. American Cancer Society Journal. 2019;171.

4. Jacinta S. Ovarian Cancer Biomarkers: Moving Forward in Early. In: Tumor Microenvironment. Springer Nature Switzerland AG; 2020. p. 356-8.

5. World Ovarian Cancer Coalition. The World ovarian cancer coalition atlas. Global trends in incidence, mortality, and survival. 2018;4-32.

6. Casanova R, Chuang A. Ovarian and Adnexal Disease. In: Beckmann and Ling's Obstetrics and Gynecology 8th. American College of Obstetricians and Gynecologists; 2019. p. 1017-9.

7. Kim B, Park Y, Kim B, Ahn HJ, Lee K-A, Chung JE, et al. Diagnostic performance of CA 125, HE4, and risk of Ovarian Malignancy Algorithm for ovarian cancer. J Clin Lab Anal. 2019 Jan;33(1):e22624.

8. Schink JC. Current initial therapy of stage III and IV ovarian cancer: challenges for managed care. Semin Oncol. 1999 Feb;26(1 Suppl 1):2-7.

9. Gentry-Maharaj A, Burnell M, Dilley J, Ryan A, Karpinskyj C, Gunu R, et al. Serum HE4 and diagnosis of ovarian cancer in postmenopausal women with adnexal masses. American Journal of Obstetrics and Gynecology. 2020 Jan;222(1):56.e1-56.e17.

10. Moore RG, McMeekin DS, Brown AK, DiSilvestro P, Miller MC, Allard WJ, et al. A novel multiple marker bioassay utilizing HE4 and CA125 for the prediction of ovarian cancer in patients with a pelvic mass. Gynecologic Oncology. 2009 Jan;112(1):40-6.

11. Karlsen MA, Høgdall EVS, Christensen IJ, Borgfeldt C, Kalapotharakos G, Zdrazilova-Dubska L, et al. A novel diagnostic index combining HE4, CA125 and age may improve triage of women with suspected ovarian cancer - An international multicenter study in women with an ovarian mass. Gynecol Oncol. 2015 Sep;138(3):640-6.

12. Yoshida A, Derchain SF, Pitta DR, De Angelo Andrade LAL, Sarian LO. Comparing the Copenhagen Index (CPH-I) and Risk of Ovarian Malignancy Algorithm (ROMA): Two equivalent ways to differentiate malignant from benign ovarian tumors before surgery? Gynecologic Oncology. 2016 Mar;140(3):481-5.

13. Dochez V, Caillon H, Vaucel E, Dimet J, Winer N, Ducarme G. Biomarkers and algorithms for diagnosis of ovarian cancer: CA125, HE4, RMI and ROMA, a review. J Ovarian Res [Internet]. 2019 Mar 27 [cited 2020 Sep 29];12. Available from: https://www.ncbi.nlm.nih.gov/pmc/articles/PMC6436208/

14. Minar L, Felsinger M, Cermakova Z, Zlamal F, Bienertova-Vasku J. Comparison of the Copenhagen Index versus ROMA for the preoperative assessment of women with ovarian tumors. Int J Gynecol Obstet. 2018 Feb;140(2):241-6. 
15. Nikolova T, Zivadinovic R, Evtimovska N, Klisarovska V, Stanojevic M, Georgievska J, et al. Diagnostic performance of human epididymis protein 4 compared to a combination of biophysical and biochemical markers to differentiate ovarian endometriosis from epithelial ovarian cancer in premenopausal women. J Obstet Gynaecol Res. 2017 Dec;43(12):1870-9.

16. Wang Z, Tao X, Ying C. CPH-I and HE4 Are More Favorable Than CA125 in Differentiating Borderline Ovarian Tumors from Epithelial Ovarian Cancer at Early Stages. Disease Markers. 2019 Oct 13;2019:1-10.

17. Høgdall E. Approaches to the detection of ovarian cancer. Scandinavian Journal of Clinical and Laboratory Investigation. 2016 Jul 13;76(sup245):S49-53.

18. Huy NVQ, Van Khoa V, Tam LM, Vinh TQ, Tung NS, Thanh CN, et al. Standard and optimal cut-off values of serum ca-125, HE4 and ROMA in preoperative prediction of ovarian cancer in Vietnam. Gynecologic Oncology Reports. 2018 Aug;25:110-4.

19. Moore RG, Miller MC, Disilvestro P, Landrum LM, Gajewski W, Ball JJ, et al. Evaluation of the diagnostic accuracy of the risk of ovarian malignancy algorithm in women with a pelvic mass. Obstet Gynecol. 2011 Aug;118(2 Pt 1):280-8.

20. Montagnana $M$, Benati $M$, Danese E. Circulating biomarkers in epithelial ovarian cancer diagnosis: from present to future perspective. Ann Transl Med. 2017 Jul;5(13).

21. Kumari S. Serum Biomarker Based Algorithms in Diagnosis of Ovarian Cancer: A Review. Indian J Clin Biochem. 2018 Oct;33(4):382-6.

\section{Tables}

\section{Table 1}

Demographic characteristics of study's subjects. 


\begin{tabular}{|c|c|c|c|c|c|c|c|}
\hline \multirow[t]{2}{*}{ Parameter } & \multicolumn{2}{|c|}{ Ovarian cancer } & \multicolumn{2}{|c|}{ Borderline } & \multicolumn{2}{|c|}{ Benign tumor } & \multirow[t]{2}{*}{$p$-value } \\
\hline & n (62) & $\%$ & n (5) & $\%$ & n (408) & $\%$ & \\
\hline \multicolumn{8}{|l|}{ Age (year) } \\
\hline$<20$ & 3 & 4.8 & 1 & 20.0 & 41 & 10.0 & $<0.0001$ \\
\hline $20-29$ & 1 & 1.6 & 1 & 20.0 & 122 & 29.9 & \\
\hline $30-39$ & 10 & 16.1 & 1 & 20.0 & 95 & 23.3 & \\
\hline $40-49$ & 11 & 17.7 & 2 & 40.0 & 74 & 18.1 & \\
\hline $50-59$ & 22 & 35.5 & - & - & 40 & 9.8 & \\
\hline$\geq 60$ & 15 & 24.2 & - & - & 36 & 8.8 & \\
\hline $\begin{array}{l}\text { Mean } \pm \text { SD } \\
(\text { Min - Max) }\end{array}$ & \multicolumn{2}{|c|}{$\begin{array}{l}50.7 \pm 15.3 \\
(11-83)\end{array}$} & \multicolumn{2}{|c|}{$\begin{array}{l}31.8 \pm 13.3 \\
(16-49)\end{array}$} & \multicolumn{2}{|c|}{$\begin{array}{l}36.0 \pm 14.9 \\
(4-86)\end{array}$} & \\
\hline \multicolumn{8}{|l|}{ Menopausal status } \\
\hline Post-menopausal & 37 & 59.7 & - & - & 68 & 16.7 & $<0.0001$ \\
\hline Pre-menopausal & 25 & 40.3 & 5 & 100.0 & 340 & 83.3 & \\
\hline \multicolumn{8}{|l|}{ Marital status } \\
\hline Single & 9 & 14.5 & - & - & 106 & 26.0 & $<0.0001$ \\
\hline Married & 53 & 85.5 & 5 & 100.0 & 302 & 74.0 & \\
\hline \multicolumn{8}{|c|}{ Number of children } \\
\hline Nulliparous & 14 & 22.6 & 1 & 20.0 & 136 & 33.3 & $>0.05$ \\
\hline Primiparous & 9 & 14.5 & 1 & 20.0 & 65 & 15.9 & \\
\hline Parity of two & 21 & 33.9 & 2 & 40.0 & 106 & 26.0 & \\
\hline Multiparous & 18 & 29.0 & 1 & 20.0 & 101 & 24.8 & \\
\hline
\end{tabular}

Abbreviations: SD, standard deviation.

\section{Table 2}

Histological classification and FIGO stages. 


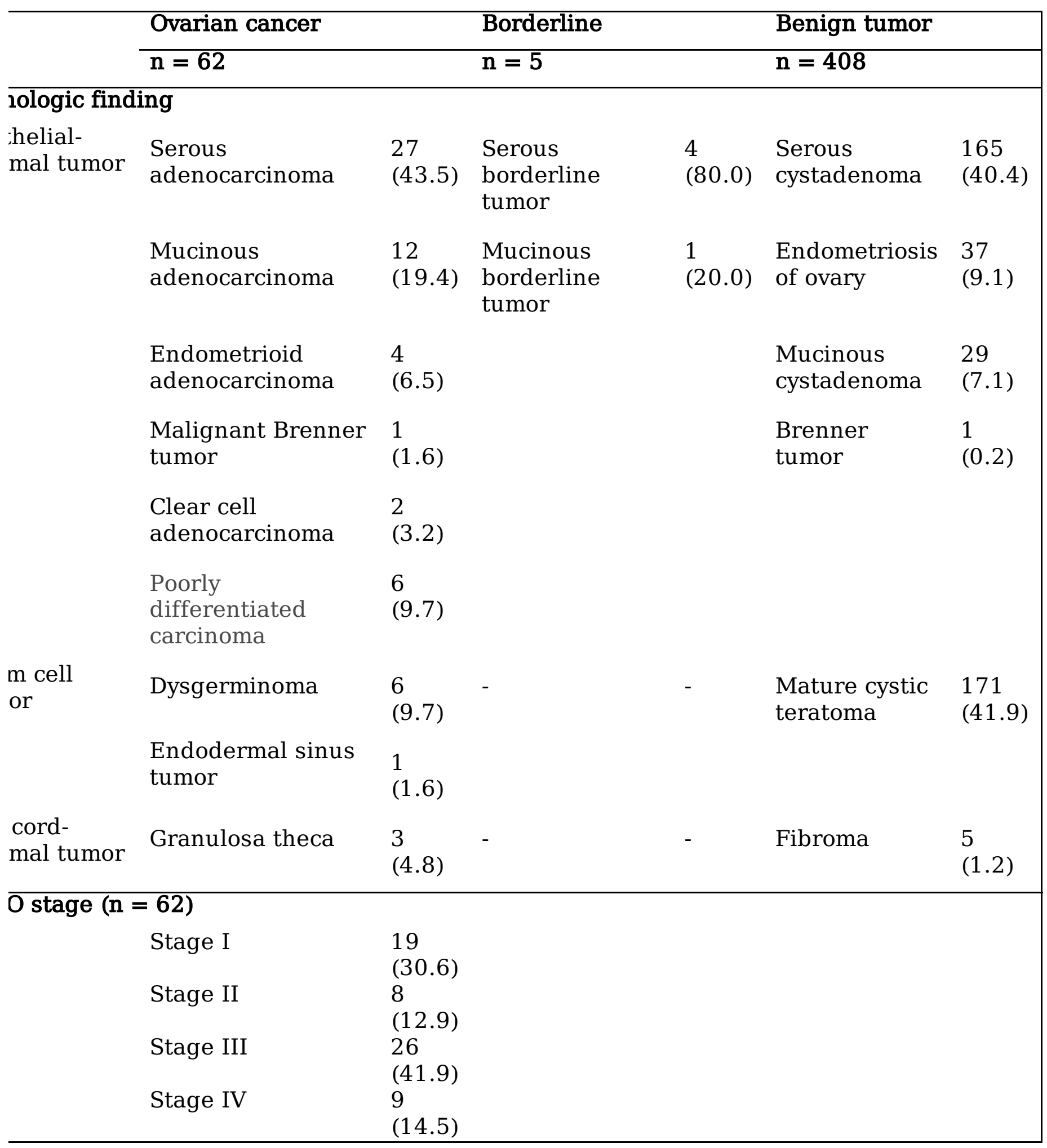

Abbreviations: FIGO, the International Federation of Gynecology and Obstetrics.

\section{Table 3}

Values of CPH-I and ROMA of study's subjects. 


\begin{tabular}{|c|c|c|c|c|c|}
\hline & \multicolumn{4}{|c|}{ Median (Q25\% - Q75\%) } & \multirow[t]{2}{*}{$P^{*}$} \\
\hline & Total & Ovarian cancer & Borderline & Benign tumor & \\
\hline Study sample & $\mathrm{n}=475$ & $\mathrm{n}=62$ & $\mathrm{n}=5$ & $\mathrm{n}=408$ & \\
\hline CPH - I & $\begin{array}{l}0.96 \\
(0.49-2.72)\end{array}$ & $\begin{array}{l}24.81 \\
(3.49-81.21)\end{array}$ & $\begin{array}{l}0.42 \\
(0.29-24.39)\end{array}$ & $\begin{array}{l}0.82 \\
(0.44-1.76)\end{array}$ & $<0.05$ \\
\hline ROMA & $\begin{array}{l}5.49 \\
(3.57-10.77)\end{array}$ & $\begin{array}{l}49.93 \\
(12.78-81.22)\end{array}$ & $\begin{array}{l}5.84 \\
(3.28-16.38)\end{array}$ & $\begin{array}{l}5.03 \\
(3.46-8.71)\end{array}$ & $<0.05$ \\
\hline Pre-menopausal & $\mathrm{n}=370$ & $\mathrm{n}=25$ & $\mathrm{n}=5$ & $\mathrm{n}=340$ & \\
\hline $\mathrm{CPH}-\mathrm{I}$ & $\begin{array}{l}0.78 \\
(0.42-1.75)\end{array}$ & $\begin{array}{l}4.87 \\
(1.49-45.72)\end{array}$ & $\begin{array}{l}0.42 \\
(0.29-24.39)\end{array}$ & $\begin{array}{l}0.72 \\
(0.41-1.43)\end{array}$ & $<0.05$ \\
\hline ROMA & $\begin{array}{l}4,63 \\
(3.19-7.22)\end{array}$ & $\begin{array}{l}12.18 \\
(6.11-62.06)\end{array}$ & $\begin{array}{l}5.84 \\
(3.28-16.38)\end{array}$ & $\begin{array}{l}4.58 \\
(3.06-6.76)\end{array}$ & $<0.05$ \\
\hline $\begin{array}{l}\text { Post- } \\
\text { menopausal }\end{array}$ & $\mathrm{n}=105$ & $\mathrm{n}=37$ & - & $\mathrm{n}=68$ & \\
\hline $\mathrm{CPH}-\mathrm{I}$ & $\begin{array}{l}3.39 \\
(1.22-16.65)\end{array}$ & $\begin{array}{l}45.49 \\
(8.35-91.62)\end{array}$ & - & $\begin{array}{l}1.49 \\
(0.87-3.65)\end{array}$ & $<0.05$ \\
\hline ROMA & $\begin{array}{l}18.71 \\
(8.93-46.47)\end{array}$ & $\begin{array}{l}72.37 \\
(37.41-95.16)\end{array}$ & - & $\begin{array}{l}10.59 \\
(7.49-18.88)\end{array}$ & $<0.05$ \\
\hline
\end{tabular}

Data are shown as median (1st to 3rd quartiles)

* Kruskal-Wallis Test

Table 4

The Validity of CPH-I and ROMA for preoperative diagnosis of ovarian cancer at optimal cut-off. 


\begin{tabular}{|c|c|c|c|c|c|}
\hline & AUC & Optimal cut-off (\%) & $\begin{array}{l}\text { Se } \\
\text { (\%) }\end{array}$ & $\begin{array}{l}\text { Sp } \\
\text { (\%) }\end{array}$ & $p$ \\
\hline \multicolumn{6}{|c|}{ Study group $(n=475)$} \\
\hline $\mathrm{CPH}-\mathrm{I}$ & 0.868 & 1.89 & 83.6 & 78.7 & $<0.05$ \\
\hline ROMA & 0.860 & 12.1 & 76.1 & 87.0 & $<0.05$ \\
\hline ROMA 1 & 0.844 & 12.1 & 74.2 & 83.5 & $<0.05$ \\
\hline ROMA 2 & 0.864 & 16.2 & 75.0 & 93.9 & $<0.05$ \\
\hline \multicolumn{6}{|c|}{ Pre-menopausal $(\mathrm{n}=370)$} \\
\hline CPH-I & 0.875 & 1.44 & 76.7 & 75.6 & $<0.05$ \\
\hline ROMA & 0.757 & 7.65 & 66.7 & 80.0 & $<0.05$ \\
\hline ROMA 1 & 0.732 & 5.67 & 84.6 & 56.6 & $<0.05$ \\
\hline ROMA 2 & 0.758 & 7.62 & 70.6 & 85.1 & $<0.05$ \\
\hline \multicolumn{6}{|c|}{ Post-menopausal $(\mathrm{n}=105)$} \\
\hline CPH-I & 0.757 & 15.4 & 72.9 & 95.6 & $<0.05$ \\
\hline ROMA & 0.927 & 43.3 & 75.7 & 98.5 & $<0.05$ \\
\hline ROMA 1 & 0.946 & 43.3 & 77.8 & 100.0 & $<0.05$ \\
\hline ROMA 2 & 0.913 & 30.2 & 78.9 & 96.2 & $<0.05$ \\
\hline
\end{tabular}

Abbreviations: CPH-I, Copenhagen Index; ROMA, Risk of Ovarian Malignancy Algorithm; AUC, Area Under the Curve; Se, Sensitivity; Sp, Specificity

\section{Table 5}

Diagnostic validity of CPH-I and ROMA from literature.

\begin{tabular}{|lllll|}
\hline Authors & \multicolumn{3}{l}{ Copenhagen Index } & ROMA \\
\cline { 2 - 5 } & AUC & Se/Sp (\%) & AUC & Se/Sp (\%) \\
\hline A. Yoshida (2016) [12] & 0.84 & $73.1 / 84.4$ & 0.82 & $71.2 / 83.5$ \\
L. Minar (2017) [14] & 0.81 & $69.0 / 85.0$ & 0.83 & $71.0 / 88.0$ \\
T. Nikolova (2017) [15] & 0.91 & $81.8 / 97.3$ & 0.90 & $90.9 / 83.8$ \\
Z. Wang (2019) [16] & 0.810 & $78.7 / 74.3$ & 0.807 & $62.9 / 88.2$ \\
Estrid Høgdall (2016) [17] & 0.960 & - & 0.954 & - \\
Nguyen Vu Quoc Huy (2018) (18) & - & - & 0.912 & $86.7 / 88.7$ \\
This study & $\mathbf{0 . 8 6 2}$ & $\mathbf{8 0 . 4 / 8 0 . 3}$ & $\mathbf{0 . 8 4 8}$ & $\mathbf{6 9 . 5 / 9 2 . 2}$ \\
\hline
\end{tabular}


Figures

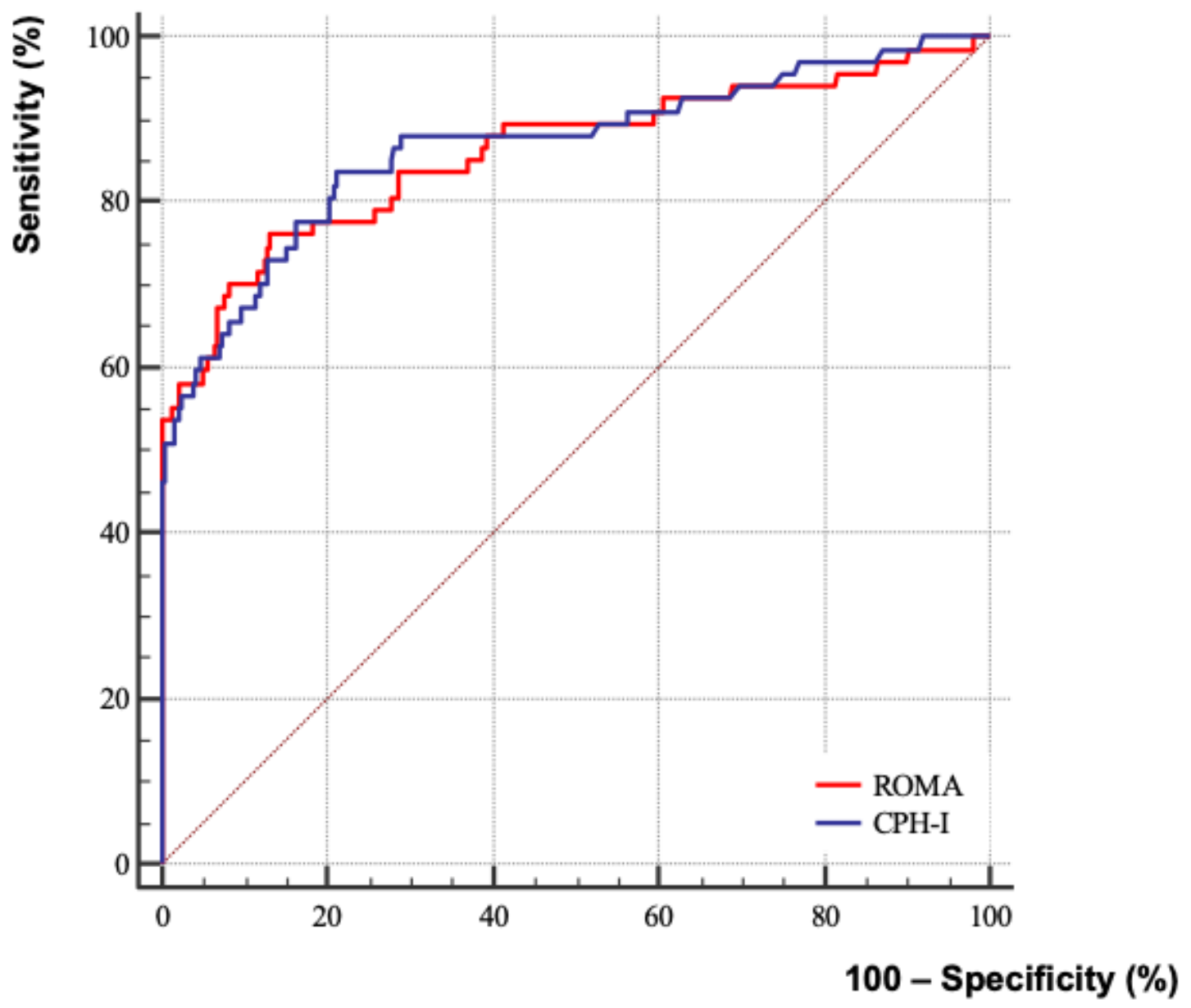

Graph 1. ROCs of $\mathrm{CPH}-\mathrm{I}$ and ROMA values in the study group.

\begin{tabular}{|lllll|}
\hline Index & $\begin{array}{l}\text { AUC } \\
(\mathbf{9 5} \% \mathrm{CI})\end{array}$ & $\begin{array}{l}\text { Se (\%) } \\
(\mathbf{9 5 \%} \mathbf{C I})\end{array}$ & $\begin{array}{l}\text { Sp (\%) } \\
(\mathbf{9 5 \%} \mathbf{C I})\end{array}$ & p-value \\
\hline CPH-I & 0.868 & 83.6 & 78.7 & \\
& $(0.833-.896)$ & $(72.5-91.5)$ & $(74.4-82.6)$ & \\
ROMA & 0.860 & 76.1 & 87.0 & 0.7374 \\
& $(0.825-0.890)$ & $(64.1-85.7)$ & $(83.4-90.1)$ & \\
\hline
\end{tabular}

Abbreviations: $\mathrm{CPH}-\mathrm{I}$, Copenhagen Index; ROMA, Risk of Ovarian Malignancy Algorithm; AUC, Area Under the Curve; Se, Sensitivity; Sp, Specificity; Cl, confidence interval;

Figure 1 
Comparison of receiver operator characteristic curves for $\mathrm{CPH}-\mathrm{I}$ and ROMA in the discrimination of benign tumors from borderline ovarian tumors and OC. 